\title{
Link State Routing Overhead in Mobile Ad Hoc Networks: A Rate-Distortion Formulation
}

\author{
Di Wang and Alhussein A. Abouzeid \\ Department of Electrical, Computer and Systems Engineering \\ Rensselaer Polytechnic Institute
}

\begin{abstract}
In this paper we use an information-theoretic formulation for characterizing the minimum routing overhead of link state routing in a mobile ad hoc network. We formulate the minimum overhead problem as a rate-distortion problem. We evaluate lower bounds on the minimum overhead incurred by maintaining link state information when link state routing protocols are designed with guaranteed delivery ratio for data packets. We also characterize the deficit caused by the routing overhead on the overall transport capacity of a mobile network. Further we derive a threshold value for the delivery error ratio, and conclude that no link state routing protocol can achieve a delivery error ratio smaller than this threshold.
\end{abstract}

\section{INTRODUCTION}

Routing in mobile ad hoc networks faces strong challenges due to dynamically changing network structure and limited communication resources. We view that the primary objective of a routing protocol is to maintain state information such that packets can be forwarded within an acceptable quality metric. The state information may be comprised of link states, node locations, velocity of nodes, queue lengths, etc. The quality metric could be minimum delay, maximum throughput, maximum lifetime or merely best effort delivery of packets.

The design of routing protocols can be viewed as choosing a subset of classes of state information about the network, and then specify when and how to collect and disseminate the chosen state information. The process of collecting state information incurs routing overhead. A number of routing protocols have been designed, and they differ from each other by selecting different subsets of classes of the state information. It may be difficult to make a comprehensive comparison among all the routing protocols, since there are many types of evaluation criteria to be used and many network scenarios to be tested. The premise of this work (as well as related prior work [1] and [2]) is that, instead of trying to find the best available protocol under certain cases, we could characterize the routing overheads incurred by a few key classes of routing protocols. The work in [1] addressed the overhead for the class of topology-based hierarchical routing protocols, while [2] considers geographic routing protocols. This paper evaluates the overhead for the class of link state routing protocols under a flat (non-hierarchical) network structure in which nodes have identical protocol responsibilities.

We present an information-theoretic framework for characterizing the minimum overhead incurred by link state routing protocols. The key departure point of this framework is to treat the link state as a random variable/process that exhibits random changes. The minimum routing overhead is the minimum amount of state information rate needed to be received by the nodes, such that the current state of the network can be identified within a certain distortion bound. This necessitates the use of information theory and rate-distortion theory as a tool for developing lower bounds on the routing overhead.

In this paper we study the class of proactive link state routing protocols, in which each node collects the link state information of the network from other nodes such that it can compute paths and thus make routing decisions immediately upon the arrival of data packets. In our analysis, the actual link status of a link and the perceived link status by an arbitrary node (say, node $k$ ) are both treated as random processes. Two types of errors associated with these two processes may occur at node $k: 1)$ the actual link status of a link is 'down' but is perceived as 'up' by $k ; 2$ ) the actual link status is 'up' but is perceived as 'down' by $k$. The link state routing overhead is the bit rate at which a node must receive link state information such that the probabilities that the two types of errors may occur are bounded. We formulate the problems of finding the minimum value of these bit rates as rate-distortion problems (see e.g. [3] for an introduction to rate-distortion theory). Using the rate-distortion formulation, we present lower bounds on minimum link state routing overhead for fixed distortions $\delta_{1}$ and $\delta_{2}$ for the two types of errors.

We further show that there is a connection between the probabilities that the two types of errors may occur, and the data packet delivery ratio $(1-\eta)$ in the network layer. This allows us to derive a lower bound on the minimum overhead for link state routing protocols with guaranteed delivery ratio $(1-\eta)$. This result is then connected with the results on the transport capacity of multi-hop wireless networks evaluated in [4] in order to characterize the effective capacity available for users. It is observed that a delivery error ratio $\eta$ cannot be achieved by any link state routing protocols if it is smaller than some threshold value $\eta^{\star}$.

In this paper we exclusively consider the scenario where the routing protocol initiates forwarding process as soon as a packet arrives at the source. Thus the capacity improvement due to node mobility (achieved at the cost of delay associated with waiting for the destination to move to a nearby location) pointed out in [5], [6] is not applicable to our work.

The class of link state routing protocols is dominant in wired networks [7]. However, when migrating from the wired to wireless, conventional link state routing protocols may incur 
large amount of overhead and thus face scalability challenges [8]. To improve the scaling properties of link state routing, a number of modified protocols have been proposed [9], [10], [11], [12], [13], [14]. Specifically, fish eye routing described in [9], [10] and [11] introduces the notion of multilevel fisheye scope to reduce routing overhead through reducing the routing packet sizes and update frequency. Optimized link state routing protocols (OLSR) [12] produce less control overhead as it forces the propagation of link state updates only at multipoint relay nodes [13]. Similarly, topology broadcast based on reverse path forwarding (TBRPF) protocols [14] reduce link state update forwarding at leaf nodes of each source tree and disseminates differential updates. To the best of our knowledge, there is still no theoretical work reported on finding the minimum link state routing overhead. The objective of this work is to fill in this gap.

The relation between this paper and the existing related work for modeling and analysis of protocol overhead can be summarized as follows. One of the earliest and pioneering works that use information theory to understand protocol overhead in communication networks can be found in [15]. Gallager [15] used an information-theoretic method to characterize the minimum amount of protocol information required to keep track of the sender, receiver and timing of messages for a simple (stationary) network model. It is pointed out that if the packet lengths are small compared to the random interarrival times, then the protocol overhead required per packet can be prohibitively high. Several relatively recent papers that use information theory to understand the effects of mobility on wireless networks can be found in [17], [18], [1] and [2]. In [17], the authors propose the entropy of link change as the metric for mobility models against which performance of wireless network protocols must be evaluated. Authors of [18] use rate-distortion theory to investigate the optimal timing for updating a certain link state metric (e.g. bandwidth) such that the update rate is minimized within a given cost constraint. One of our prior works [1] uses an information-theoretic approach to characterize the minimum routing overhead and memory requirements of hierarchical routing protocols for ad hoc networks. Our work shares a similar flavor with another one of our prior work [2], in which the family of geographic routing protocols are studied and rate-distortion lower bounds on geographic routing overhead are derived.

Compared to [1], [2] and other related works, our main contributions may be summarized as follows:

1) We present an information-theoretic formulation for evaluating routing overhead incurred by link state routing. As compared to our prior work [1], apart from the different network structures studied (flat and hierarchical), the routing protocol model used in [1] specifies the exact mechanisms about when and how to update network state information. Such specifications are not used in this work and thus this formulation is more general.

2) We evaluate lower bounds for the minimum rate at which a node must receive link state information such that data

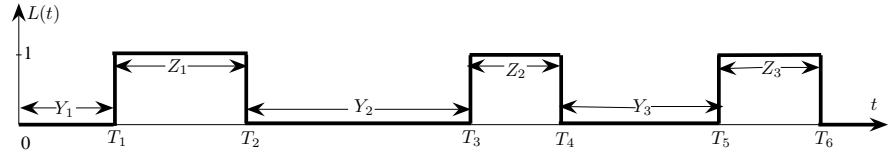

Fig. 1. An illustration of the relationship between random variables $L(t)$, $Y_{m}$ and $Z_{m}$ associated with node pair $(i, j)$.

packets can be delivered with guaranteed delivery ratio.

3) We characterize the effective transport capacity of a mobile ad hoc network after taking into account the minimum routing overhead incurred by reliable link state routing.

4) We derive the threshold value $\eta^{\star}$ for the delivery error ratio, and point out that no link state routing protocols can achieve a delivery error ratio smaller than this threshold.

The rest of this paper is organized as follows. The network and routing protocol models are presented in Section II. The rate-distortion formulation and the evaluation of a lower bound on the minimum routing overhead are presented in Section III. With the results in Section III, the rate-distortion lower bounds on the overheads of link state routing protocols with guaranteed delivery ratio are derived in Section IV-A, and a discussion of the capacity deficit caused by routing overheads is presented in Section IV-B. We present conclusions and future research directions in Section V.

\section{MODEL}

\section{A. Network Model}

The ad hoc network consists of $n$ mobile nodes all lying in a torus of unit area. The location of node $i$ at time $t$ is given by $X_{i}(t), i=1, \ldots, n$. The process $\left\{X_{i}(\cdot)\right\}$ is stationary and ergodic with stationary distribution uniform on the area of interest; moreover, the trajectories of different nodes are independent and identically distributed (i.i.d.). Each node is equipped with an omnidirectional antenna and the transmission radius is given by $r$. It is assumed that $n \pi r^{2}>1$, which is a necessary condition for network connectivity.

Let the random variable $L_{i j}(t) \in\{0,1\}$ denote the link status between any two nodes $i$ and $j$ at time $t$, where 1(0) represents the link state is up (down). Formally,

$$
L_{i j}(t)= \begin{cases}1, & \text { if }\left|X_{i}(t)-X_{j}(t)\right| \leq r, \\ 0, & \text { otherwise }\end{cases}
$$

Note that $L_{i j}(t)$ is induced by $X_{i}(t)$ and $X_{j}(t)$, thus it is also a stationary ergodic process. For the sake of notation simplicity, in the rest of this paper we will use $L(t)$ instead of $L_{i j}(t)$ if there is no ambiguity.

In order to facilitate the analysis, the following assumptions regarding $\{L(\cdot)\}$ are made:

Assumption 1: All the processes $\left\{L_{i j}(\cdot) \mid i \neq j, 1 \leq i, j \leq\right.$ $n\}$ are assumed to be mutually independent. Although this assumption may not be true in general, theoretical analysis in [1, Section III.C.4] and simulation studies presented in [16, 


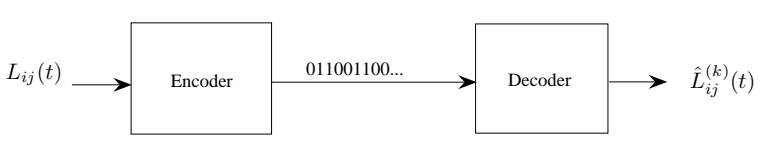

Fig. 2. An illustration of encode-transmit-receive-and-decode procedure for node $k$ to monitor the status of link $(i, j)$

Section 9] show that the dependency between two neighboring links is weak and can be negligible.

Assumption 2: The random process $L(\cdot)$ is modeled as an alternating renewal process ([19]), which is elaborated as follows. Let $\left\{Y_{m}\right\}_{m=1}^{\infty}$ and $\left\{Z_{m}\right\}_{m=1}^{\infty}$ denote the sequences of successive 'up' and 'down' time durations of $L(t)$ (the relationship between $L(t), Y_{m}$ and $Z_{m}$ is briefly illustrated by Fig. 1). Both the sequence $\left\{Y_{m}\right\}_{m=1}^{\infty}$ and the sequence $\left\{Z_{m}\right\}_{m=1}^{\infty}$ are assumed to be independent and identically distributed (i.i.d.) with probability density functions (pdf) $f_{Y}(\cdot)$ and $f_{Z}(\cdot)$, respectively. Moreover, we assume that $\left\{Y_{m}\right\}_{m=1}^{\infty}$ and $\left\{Z_{m}\right\}_{m=1}^{\infty}$ are independent from each other. The expected values of $Y_{m}$ and $Z_{m}$ are denoted by $\mu_{Y}$ and $\mu_{Z}$, respectively. For the convenience of further analysis, we define $u$ as

$$
u=\mu_{Y}+\mu_{Z}
$$

\section{B. Link State Routing Protocol Model}

A link state routing protocol requires each node to collect link state information from other nodes such that it can compute a path to any potential destination node in the network. The process of collecting link state information produces a routing overhead. Specifically, the link state information of any given node pair $(i, j)(i \neq j)$ may be encoded and sent out as control packets by the routing protocol, and then may be received and decoded by some node $k(k=1, \ldots, n)$ in order to allow node $k$ to monitor the link status of $(i, j)$. The link state routing overhead associated with node $k$ is thus formed by bit streams of all such control packets received by node $k$, for any given $k=1, \ldots, n$.

This encode-transmit-receive-and-decode procedure is illustrated by Fig. 2. The link status of $(i, j)$, which is represented by $L(t)$, is encoded by the link state routing protocol and then sent to node $k$ such that $k$ is able to reproduce it as $\hat{L}_{i j}^{(k)}(t)$ (for simplicity, we will use $\hat{L}(t)$ instead in the rest of this paper). Here we make two assumptions as follows:

Assumption 3: It is assumed that the perceived link status $\hat{L}(t)$ is updated and maintained based on the link state information collected by $k$ from outside only. This means that node $k$ does not make its own decisions to determine $\hat{L}(t)$ at any given time $t$ (e.g., no prediction based schemes are used); link status is maintained and updated according to the received control packets only.

Assumption 4: It is assumed that the positions of node $i$ and $j$ do not change significantly during the encode-transmitreceive-and-decode procedure described above. In other words the time scale of forwarding a packet is much smaller than that required for a significant change in position.
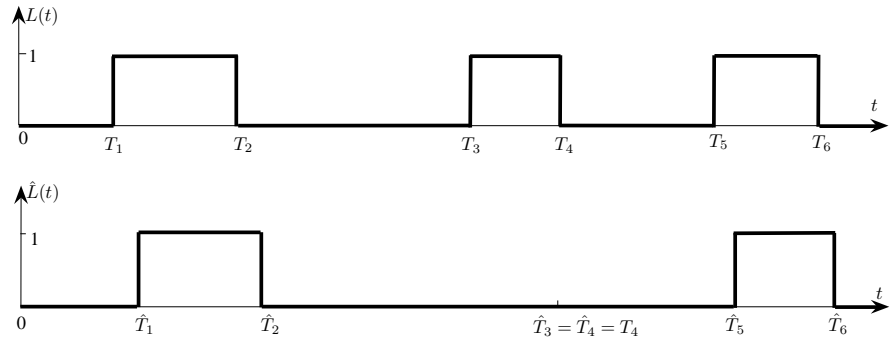

Fig. 3. An example of $(i, j)$ 's link status $L(t)$ associated with the sequence $\left\{T_{m}\right\}$, and the perceived link status $\hat{L}(t)$ at node $k$ associated with the sequence $\left\{\hat{T}_{m}\right\}$.

\section{OVERHEAD ANALYSis}

For any given nodes $i, j$ and $k$, there are basically two types of errors associated with $(i, j)$ 's link status $L(t)$ and the perceived link status $\hat{L}(t)$ by $k: 1)$ type-1 error is the one that happens at time $t$ if $L(t)=0$ and $\hat{L}(t)=1$; 2) type-2 error is the one that happens at time $t$ if $L(t)=1$ and $\hat{L}(t)=0$. In this section we evaluate a lower bound on the minimum bit rate at which a node must receive link state information, such that the time portions of these two types of errors are bounded from above by $\delta_{1}$ and $\delta_{2}$, respectively.

\section{A. Notation and Rate-Distortion Formulation}

Definition 1: $S_{1}$ is the set of time instants at which type-1 error happens during the time interval $[0, w]$, i.e., $S_{1}=\{t \mid 0 \leq$ $t \leq w, L(t)=0, \hat{L}(t)=1\}$. Similarly, $S_{2}$ is the set of time instants at which type-2 error happens during the time interval $[0, w]$, i.e., $S_{2}=\{t \mid 0 \leq t \leq w, L(t)=1, \hat{L}(t)=0\}$.

Definition 2: $D_{w}^{(1)}(L, \hat{L})$ is the time portion of type-1 error in the link state information of node pair $(i, j)$ at node $k$ over a time period $[0, w]$, i.e.,

$$
D_{w}^{(1)}(L, \hat{L})=\frac{1}{w} \int_{t \in S_{1}}|L(t)-\hat{L}(t)| d t
$$

Similarly, $D_{w}^{(2)}(L, \hat{L})$ is the time portion of type- 2 error in the link state information of node pair $(i, j)$ at node $k$ over a time period $[0, w]$, i.e.,

$$
D_{w}^{(2)}(L, \hat{L})=\frac{1}{w} \int_{t \in S_{2}}|L(t)-\hat{L}(t)| d t
$$

Definition 3: $\left\{T_{m}\right\}_{m=1}^{\infty}$ is the sequence of time instants at which the link status of $(i, j)$ changes (i.e. the value of $L(t)$ changes).

Based on the Assumption 3 and Assumption 4 (made in Section II-B), it can be observed that every change in $\hat{L}(t)$ follows after a change in $L(t)$ at some time instant $T_{m}$ and is followed by a change in $L(t)$ at the time instant $T_{m+1}$. And there is at most one change in $\hat{L}(t)$ between $T_{m}$ and $T_{m+1}$ for any given $m$. Let $\hat{T}_{m}$ denote such a change in $\hat{L}(t)$. Fig. 3 illustrates an example of $L(t)$ and $\hat{L}(t)$. It shows that $\hat{L}(t)$ changes its value at $\hat{T}_{1}$ and $\hat{T}_{2}$, and has $T_{1} \leq \hat{T}_{1} \leq T_{2}$ and $T_{2} \leq \hat{T}_{2} \leq T_{3}$. It can be noted that $\hat{T}_{m}$ is not clearly defined for some values of $m$ ( $m=3,4$ for the example shown in 
Fig. 3). To make the definition of $\hat{T}_{m}$ complete, we define $\hat{T}_{3}=\hat{T}_{4}=T_{4}$ in the example illustrated by Fig. 3. Formally, we have the following definition for $\hat{T}_{m}$.

Definition 4: Let $\left\{\hat{T}_{m}\right\}_{m=1}^{\infty}$ denote a sequence of time instants which is defined according to the following two cases:

1) If $\hat{L}(t)$ is not constant over $\left(T_{m}, T_{m+1}\right)$, i.e., there exists $c \in\{0,1\}$ and $\tau \in\left(T_{m}, T_{m+1}\right)$ such that

$$
\hat{L}(t)= \begin{cases}c, & \forall t \in\left(T_{m}, \tau\right) ; \\ 1-c, & \forall t \in\left(\tau, T_{m+1}\right),\end{cases}
$$

then let

$$
\hat{T}_{m}:=\tau
$$

2) If $\hat{L}(t)$ is constant over $\left(T_{m}, T_{m+1}\right)$, i.e., $\hat{L}(t)=c \in$ $\{0,1\}$, for any $t \in\left(T_{m}, T_{m+1}\right)$, then let

$$
\hat{T}_{m}:= \begin{cases}T_{m}, & \text { if } L(t)=c, \forall t \in\left(T_{m}, T_{m+1}\right) \\ T_{m+1}, & \text { if } L(t) \neq c, \forall t \in\left(T_{m}, T_{m+1}\right) .\end{cases}
$$

Thus we have a sequence $\left\{\hat{T}_{m}: m=1,2, \ldots\right\}, \hat{T}_{0}=0$, where $\hat{T}_{m}$ denotes the time instant at which $\hat{L}(t)$ changes its value, and the following inequality

$$
T_{m} \leq \hat{T}_{m} \leq T_{m+1}
$$

holds for $m=0,1,2, \ldots$.

Definition 5: Let $N(w)$ denote the number of changes in $L(t)$ during the time interval $[0, w]$. Formally,

$$
N(w)=\sup \left\{m \mid T_{m} \leq w\right\}
$$

Similarly, $N^{\prime}(w)$ is defined as

$$
N^{\prime}(w)=\sup \left\{m \mid \hat{T}_{m} \leq w\right\}
$$

(For the sake of simplicity, we will use $N$ and $N^{\prime}$ instead of $N(w)$ and $N^{\prime}(w)$ in the rest of this paper.)

By Definition 3-5, it is readily known that

$$
N-1 \leq N^{\prime} \leq N
$$

Then according to the network model described in Section II$\mathrm{B}$, the average rate of $(i, j)$ 's link status changes is given by

$$
\lim _{w \rightarrow \infty} \frac{N}{w}=\lim _{w \rightarrow \infty} \frac{N^{\prime}}{w}=\frac{2}{\mu_{Y}+\mu_{Z}}=\frac{2}{u}
$$

Definition 6: $T^{N}$ and $\hat{T}^{N^{\prime}}$ are defined as random vectors such that $T^{N}=\left[T_{1}, T_{2}, \ldots, T_{N}\right]$ and $\hat{T}^{N^{\prime}}=\left[\hat{T}_{1}, \hat{T}_{2}, \ldots, \hat{T}_{N^{\prime}}\right]$. Define $T^{0}:=T_{0}=0$ and $\hat{T}^{0}:=\hat{T}_{0}=0$ for the trivial case $N=0$.

Definition 7: $\mathcal{T}^{N}$ and $\hat{\mathcal{T}}^{N^{\prime}}$ are defined as the sets of all possible vectors $T^{N}$ and $\hat{T}^{N^{\prime}}$, respectively.

Definition 8: $P_{w}\left[t^{N} ; \hat{t}^{N^{\prime}}\right]$ denotes the joint probability density function (pdf) of random vectors $T^{N}$ and $\hat{T}^{N^{\prime}}$, with $t^{N} \in \mathcal{T}^{N}$ and $\hat{t}^{N^{\prime}} \in \hat{\mathcal{T}}^{N^{\prime}}$.

Definition 9: $\bar{D}_{w}^{(1)}$ is the expected value of the time portion of type- 1 error over the time period $[0, w]$, i.e.,

$$
\bar{D}_{w}^{(1)}=E\left[D_{w}^{(1)}\right]=\int_{t^{N} \in \mathcal{T}^{N}, \hat{t}^{N^{\prime}} \in \hat{\mathcal{T}}^{N^{\prime}}} P_{w}\left[t^{N} ; \hat{t}^{N^{\prime}}\right] D_{w}^{(1)} d t^{N} d \hat{t}^{N^{\prime}}
$$

Similarly, $\bar{D}_{w}^{(2)}$ is the expected value of the time portion of type- 2 error over the time period $[0, w]$, i.e.,

$$
\bar{D}_{w}^{(2)}=E\left[D_{w}^{(2)}\right]=\int_{t^{N} \in \mathcal{T}^{N}, \hat{t}^{N^{\prime}} \in \hat{\mathcal{T}}^{N^{\prime}}} P_{w}\left[t^{N} ; \hat{t}^{N^{\prime}}\right] D_{w}^{(2)} d t^{N} d \hat{t}^{N^{\prime}}
$$

Definition 10: $\mathcal{P}_{w}\left(\delta_{1}, \delta_{2}\right)$ is defined as the family of pdfs $P_{w}\left[t^{N} ; \hat{t}^{N^{\prime}}\right]$ such that the following inequalities hold:

$$
\left\{\begin{array}{l}
T_{m} \leq \hat{T}_{m} \leq T_{m+1} \\
\bar{D}_{w}^{(1)} \leq \delta_{1} \\
\bar{D}_{w}^{(2)} \leq \delta_{2}
\end{array}\right.
$$

Now we will present a rate-distortion based formulation to find the minimum bit rate required to represent the link state information such that the inequalities in (15) are satisfied.

Definition 11: $R_{w}\left(\delta_{1}, \delta_{2}\right)$ is defined as the rate-distortion function associated with the time interval $[0, w]-$ the minimum bit rate at which a node must receive link state information such that the inequalities in (15) are satisfied. According to [3], $R_{w}\left(\delta_{1}, \delta_{2}\right)$ is given by

$$
R_{w}\left(\delta_{1}, \delta_{2}\right)=\min _{P_{w} \in \mathcal{P}_{w}\left(\delta_{1}, \delta_{2}\right)} \frac{1}{w} I\left(T^{N} ; \hat{T}^{N^{\prime}}\right)
$$

where $I\left(T^{N} ; \hat{T}^{N^{\prime}}\right)$ is the mutual information between $T^{N}$ and $\hat{T}^{N^{\prime}}$.

The minimum rate (in bits/sec) at which a node must receive link state information of $(i, j)$ such that a large fraction of packets are delivered, represented by $R\left(\delta_{1}, \delta_{2}\right)$, is given by

$$
R\left(\delta_{1}, \delta_{2}\right)=\lim _{w \rightarrow \infty} R_{w}\left(\delta_{1}, \delta_{2}\right)
$$

In order to facilitate the analysis in the following section, we define another two rate-distortion functions as follows.

Consider the i.i.d. sequence $\left\{Y_{m}\right\}$ drawn according to the pdf $f_{Y}(y), y \in \mathcal{Y}$. Let $\left\{\hat{Y}_{m}\right\}$ denote the reconstruction of $\left\{Y_{m}\right\}$. We assume that for any given $m$, the following reconstruction constraint is satisfied:

$$
\hat{Y}_{m} \geq Y_{m}
$$

Let $f_{Y, \hat{Y}}(y, \hat{y})$ be the joint pdf of $Y$ and $\hat{Y},(y, \hat{y}) \in \mathcal{Y} \times \hat{\mathcal{Y}}$. The distortion measure, $d: \mathcal{Y} \times \hat{\mathcal{Y}} \rightarrow \mathbb{R}^{+}$, is defined as

$$
d(y, \hat{y})=\hat{y}-y
$$

Let $\mathcal{F}^{(Y)}(\epsilon)$ denote the family of the joint pdf $f_{Y, \hat{Y}}(y, \hat{y})$ satisfying

$$
f_{Y, \hat{Y}}(y, \hat{y})=0, \text { for } y>\hat{y}
$$

and

$$
E[d(y, \hat{y})]=\int_{y} \int_{\hat{y}}(\hat{y}-y) f_{Y, \hat{Y}}(y, \hat{y}) d y d \hat{y} \leq \epsilon .
$$

Thus the rate-distortion function for $Y$ is defined as follows.

Definition 12: $R^{(Y)}(\epsilon)$ is the rate-distortion function for source $Y$, with reconstruction constraint (18) and distortion measure (19). According to [3], $R^{(Y)}(\epsilon)$ is given by

$$
R^{(Y)}(\epsilon)=\min _{f_{Y, \hat{Y}}(y, \hat{y}) \in \mathcal{F}^{(Y)}(\epsilon)} I(Y ; \hat{Y})
$$


Similarly, we define $R^{(Z)}(\epsilon)$ as the rate-distortion function for the i.i.d. sequence $\left\{Z_{m}\right\}$ drawn according to the pdf $f_{Z}(z)$ with the same reconstruction constraint and distortion measure as in Definition 12.

\section{B. Link State Routing Overhead}

Lemma 1: The mutual information between $T^{N}$ and $\hat{T}^{N^{\prime}}$ satisfies the following relationship

$$
\begin{aligned}
& \inf _{P_{w} \in \mathcal{P}_{w}\left(\delta_{1}, \delta_{2}\right)} I\left(T^{N} ; \hat{T}^{N^{\prime}}\right) \\
& \geq\left\lfloor\frac{N^{\prime}}{2}\right\rfloor R^{(Z)}\left(\frac{w \delta_{1}}{\left\lfloor\frac{N^{\prime}}{2}\right\rfloor}\right)+\left\lfloor\frac{N^{\prime}}{2}\right\rfloor R^{(Y)}\left(\frac{w \delta_{2}}{\left\lfloor\frac{N^{\prime}}{2}\right\rfloor}\right)
\end{aligned}
$$

Proof: Note that

$$
\begin{aligned}
& I\left(T^{N} ; \hat{T}^{N^{\prime}}\right)-I\left(T^{N^{\prime}} ; \hat{T}^{N^{\prime}}\right) \\
= & \begin{cases}I\left(T_{N} ; \hat{T}^{N^{\prime}} \mid T^{N^{\prime}}\right)(\geq 0), & \text { if } N=N^{\prime}+1 ; \\
0, & \text { otherwise. }\end{cases}
\end{aligned}
$$

Then only need to show that $I\left(T^{N^{\prime}} ; \hat{T}^{N^{\prime}}\right)$ is no less than the right-hand side of equation (23).

When $N^{\prime}$ is odd, i.e., $N^{\prime}=2 M+1$ and $M \in \mathbb{N}$, we have

$$
\begin{aligned}
& I\left(T^{N^{\prime}} ; \hat{T}^{N^{\prime}}\right) \\
= & I\left(T^{2 M}, T_{2 M+1} ; \hat{T}^{2 M}, \hat{T}_{2 M+1}\right) \\
= & I\left(T^{2 M} ; \hat{T}^{2 M}, \hat{T}_{2 M+1}\right)+I\left(T_{2 M+1} ; \hat{T}^{2 M}, \hat{T}_{2 M+1} \mid T^{2 M}\right) \\
\geq & I\left(T^{2 M} ; \hat{T}^{2 M}, \hat{T}_{2 M+1}\right) \\
= & I\left(T^{2 M} ; \hat{T}^{2 M}\right)+I\left(T^{2 M} ; \hat{T}_{2 M+1} \mid \hat{T}^{2 M}\right) \\
\geq & I\left(T^{2 M} ; \hat{T}^{2 M}\right)
\end{aligned}
$$

Thus only need to show that (23) is true for $N^{\prime}$ is even, i.e., $N^{\prime}=2 M$ and $M \in \mathbb{N}$.

Using the standard definition of mutual information we get

$$
I\left(T^{2 M} ; \hat{T}^{2 M}\right)=h\left(T^{2 M}\right)-h\left(T^{2 M} \mid \hat{T}^{2 M}\right)
$$

where $h(\cdot)$ denotes the differential entropy.

Then we consider $h\left(T^{2 M} \mid \hat{T}^{2 M}\right)$. Without loss of generality, we assume that $L(0)=0$, i.e. $T_{1}=Y_{1}$. Thus

$$
\begin{aligned}
Y_{m} & =T_{2 m-1}-T_{2 m-2} \\
Z_{m} & =T_{2 m}-T_{2 m-1}
\end{aligned}
$$

Then

$$
\begin{aligned}
h\left(T^{2 M} \mid \hat{T}^{2 M}\right)= & \sum_{m=1}^{2 M} h\left(T_{m} \mid T^{m-1}, \hat{T}^{2 M}\right) \\
\leq & \sum_{m=1}^{2 M} h\left(T_{m} \mid T_{m-1}, \hat{T}_{m}\right) \\
= & \sum_{m=1}^{2 M} h\left(T_{m}-T_{m-1} \mid T_{m-1}, \hat{T}_{m}\right) \\
= & \sum_{m=1}^{M}\left(h\left(Y_{m} \mid T_{2 m-2}, \hat{T}_{2 m-1}\right)\right. \\
& \left.+h\left(Z_{m} \mid T_{2 m-1}, \hat{T}_{2 m}\right)\right)
\end{aligned}
$$

Equation (30) follows from (29) since conditioning does not increase entropy, and (31) follows from (30) since
$h\left(T_{m} \mid T_{m-1}, \hat{T}_{m}\right)$ is already conditioned on $T_{m-1}$, subtracting it from $T_{m}$ is similar to translating the random variable by a scalar. Equation (32) follows directly from (31), (27) and (28).

Define a random vector $U_{m}$ such that

$$
U_{m}=\hat{T}_{m}-T_{m-1}
$$

Since $U_{m}$ depends only on $\hat{T}_{m}$ and $T_{m-1}$, we have

$$
\begin{aligned}
h\left(Y_{m} \mid T_{2 m-2}, \hat{T}_{2 m-1}\right) & =h\left(Y_{m} \mid T_{2 m-2}, \hat{T}_{2 m-1}, U_{2 m-1}\right) \\
& \leq h\left(Y_{m} \mid U_{2 m-1}\right) ;
\end{aligned}
$$

similarly we can get $h\left(Z_{m} \mid T_{2 m-1}, \hat{T}_{2 m}\right) \leq h\left(Z_{m} \mid U_{2 m}\right)$.

Then we get the following upper bound for $h\left(T^{2 M} \mid \hat{T}^{2 M}\right)$ :

$$
h\left(T^{2 M} \mid \hat{T}^{2 M}\right) \leq \sum_{m=1}^{M}\left(h\left(Y_{m} \mid U_{2 m-1}\right)+h\left(Z_{m} \mid U_{2 m}\right)\right)
$$

Now consider $h\left(T^{2 M}\right)$. Since $Y_{1}, \ldots, Y_{m}, \ldots, Z_{1}, \ldots, Z_{m}, \ldots$ are independent, we have

$$
h\left(T^{2 M}\right)=\sum_{m=1}^{M}\left(h\left(Y_{m}\right)+h\left(Z_{m}\right)\right)
$$

By combining (26), (34) and (35), we get

$$
\begin{array}{cl}
I\left(T^{2 M} ; \hat{T}^{2 M}\right) \geq \sum_{m=1}^{M}\left(I\left(Y_{m} ; U_{2 m-1}\right)+I\left(Z_{m} ; U_{2 m}\right)\right) \\
\text { Define } \quad \begin{aligned}
\rho_{m}^{(Y)} & =E\left[U_{2 m-1}-Y_{m}\right] \\
& \rho_{m}^{(Z)}=E\left[U_{2 m}-Z_{m}\right]
\end{aligned}
\end{array}
$$

By equation (33) it is easy to verify that $U_{2 m-1}-Y_{m} \geq 0$ and $U_{2 m}-Z_{m} \geq 0$. Thus by Definition 12 we have

$$
\begin{aligned}
I\left(Y_{m} ; U_{2 m-1}\right) & \geq R^{(Y)}\left(\rho_{m}^{(Y)}\right) \\
I\left(Z_{m} ; U_{2 m}\right) & \geq R^{(Z)}\left(\rho_{m}^{(Z)}\right)
\end{aligned}
$$

From (3), (8) and (13) we have

$$
\begin{aligned}
\bar{D}_{w}^{(1)} & =E\left[\frac{1}{w} \int_{t \in S_{1}}|L(t)-\hat{L}(t)| d t\right] \\
& \geq \frac{1}{w} E\left[\sum_{m=1}^{M}\left(U_{2 m}-Z_{m}\right)\right] \\
& =\frac{1}{w} \sum_{m=1}^{M} \rho_{m}^{(Z)}
\end{aligned}
$$

Similarly we can get $\bar{D}_{w}^{(2)}=\frac{1}{w} \sum_{m=1}^{M} \rho_{m}^{(Y)}$.

By substituting (39) and (40) in (36) and noticing the fact that rate-distortion functions $R^{(Y)}(\cdot)$ and $R^{(Z)}(\cdot)$ are convex and non-increasing, we have

$$
\begin{aligned}
& I\left(T^{2 M} ; \hat{T}^{2 M}\right) \\
\geq & \sum_{m=1}^{M} R^{(Y)}\left(\rho_{m}^{(Y)}\right)+\sum_{m=1}^{M} R^{(Z)}\left(\rho_{m}^{(Z)}\right) \\
\geq & M R^{(Y)}\left(\frac{1}{M} \sum_{m=1}^{M} \rho_{m}^{(Y)}\right)+M R^{(Z)}\left(\frac{1}{M} \sum_{m=1}^{M} \rho_{m}^{(Z)}\right) \\
\geq & M R^{(Y)}\left(\frac{w}{M} \bar{D}_{w}^{(2)}\right)+M R^{(Z)}\left(\frac{w}{M} \bar{D}_{w}^{(1)}\right) \\
\geq & M R^{(Z)}\left(\frac{w \delta_{1}}{M}\right)+M R^{(Y)}\left(\frac{w \delta_{2}}{M}\right)
\end{aligned}
$$


Hence we have proved this lemma.

The following lemma provides a lower bound on the minimum rate (in bits/sec) at which a node $k$ must receive link state information about an arbitrary node pair $(i, j)$.

Lemma 2: Under the distortion criterion parameterized by $\delta_{1}$ and $\delta_{2}$, the lower bound on the minimum rate (in bits/sec) at which a node $k$ must receive link state information of an arbitrary node pair $(i, j)$ is given by

$$
R\left(\delta_{1}, \delta_{2}\right) \geq \frac{1}{u}\left(h(Y)+h(Z)+\log _{2} \frac{1}{e^{2} u^{2} \delta_{1} \delta_{2}}\right)
$$

where $u$ is defined in equation (2).

Proof: From Equation (12), Lemma 1 and the definitions of rate-distortion functions (16) and (17), it follows that

$$
\begin{aligned}
& R\left(\delta_{1}, \delta_{2}\right)=\lim _{w \rightarrow \infty} R_{w}\left(\delta_{1}, \delta_{2}\right) \\
\geq & \lim _{w \rightarrow \infty}\left(\frac{\left\lfloor\frac{N^{\prime}}{2}\right\rfloor}{w} R^{(Z)}\left(\frac{w \delta_{1}}{\left\lfloor\frac{N^{\prime}}{2}\right\rfloor}\right)+\frac{\left\lfloor\frac{N^{\prime}}{2}\right\rfloor}{w} R^{(Y)}\left(\frac{w \delta_{2}}{\left\lfloor\frac{N^{\prime}}{2}\right\rfloor}\right)\right) \\
= & \frac{R^{(Z)}\left(u \delta_{1}\right)+R^{(Y)}\left(u \delta_{2}\right)}{u}
\end{aligned}
$$

According to Definition 12, the rate-distortion function $R^{(Y)}(\epsilon)$ is given by

$$
R^{(Y)}(\epsilon)=\min _{f_{Y, \hat{Y}}(y, \hat{y}) \in \mathcal{F}^{(Y)}(\epsilon)} I(Y ; \hat{Y})
$$

Let $\operatorname{Expo}(\varphi)$ denote the exponential distribution with mean $\varphi$. Then

$$
\begin{aligned}
I(Y ; \hat{Y}) & =h(Y)-h(Y \mid \hat{Y}) \\
& =h(Y)-h(\hat{Y}-Y \mid \hat{Y}) \\
& \geq h(Y)-h(\hat{Y}-Y) \\
& \geq h(Y)-h(\operatorname{Expo}(E[\hat{Y}-Y])) \\
& \geq h(Y)-\log _{2}(e \epsilon)
\end{aligned}
$$

Here equation (47) follows from (46) since for a nonnegative random variable with fixed mean, exponential distribution maximizes the differential entropy. (48) follows from (47) since $f_{Y, \hat{Y}}(y, \hat{y}) \in \mathcal{F}^{(Y)}(\epsilon)$. Thus

$$
R^{(Y)}(\epsilon) \geq h(Y)-\log _{2}(e \epsilon)
$$

Similarly

$$
R^{(Z)}(\epsilon) \geq h(Z)-\log _{2}(e \epsilon)
$$

By substituting (49) and (50) in (44) we get (43) and hence have proved this lemma.

Since there are totally $\frac{n(n-1)}{2}$ node pairs and based on Assumption 1, we get the following theorem that provides a lower bound on the minimum rate at which a node must receive link state information from the entire network.

Theorem 1: Under the distortion criterion parameterized by $\delta_{1}$ and $\delta_{2}$, the lower bound on the minimum rate (in bits/sec) at which a node must receive link state information from the entire network, denoted by $\Phi\left(\delta_{1}, \delta_{2}\right)$, is given by

$$
\Phi\left(\delta_{1}, \delta_{2}\right) \geq \frac{n(n-1)}{2 u}\left(h(Y)+h(Z)+\log _{2} \frac{1}{e^{2} u^{2} \delta_{1} \delta_{2}}\right)
$$

Theorem 1 implies that, apart from the distortion-related parameters $\delta_{1}$ and $\delta_{2}$, mobility patterns impose a major impact on the minimum link state update rate, since it largely depends on $h(Y), h(Z)$ and $u$.

\section{Routing Overhead Under Markovian Mobility Pattern}

Now we consider a special case for the mobility pattern, where we assume that both the up-time $Z$ and down-time $Y$ for each link follow exponential distributions. This assumption implies that the process $\{L(t)\}$, which describes the link status changes, is a two-state Markov process. This allows us to derive closed-form results for the lower bound on $\Phi$, and therefore we may gain some insights without involving with complicated analysis.

Since both $Y$ and $Z$ follow exponential distributions, then

$$
\begin{aligned}
& h(Y)=\log _{2}\left(e \mu_{Y}\right) \\
& h(Z)=\log _{2}\left(e \mu_{Z}\right)
\end{aligned}
$$

Since the process $L(t)$ is a stationary ergodic process (see Section II-A), then it is readily known that

$$
\operatorname{Pr}[(i, j) \text { 's link status is up }]=\frac{\mu_{Z}}{\mu_{Y}+\mu_{Z}}=\pi r^{2}
$$

By substituting (2), (52), (53) and (54) in (51), we get the following corollary.

Corollary 1: When the network is exhibiting Markovian link status changes, the lower bound on the minimum rate (in bits/sec) at which a node must receive link state information from the entire network under the distortion criterion parameterized by $\delta_{1}$ and $\delta_{2}$, is given by

$$
\Phi \geq \frac{n(n-1)}{2 u} \log _{2} \frac{\pi r^{2}\left(1-\pi r^{2}\right)}{\delta_{1} \delta_{2}}
$$

\section{Practical Implications}

In the previous section, we evaluated the lower bound on the minimum rate of link state routing overhead such that the error in the link state information available at each node is bounded. In this section, we will show that this result can be applied to capture the interplay between control and data traffic, i.e., the tradeoff between the overhead incurred by control packets and the delivery ratio for data packets. Then we characterize the deficit caused by the routing overhead on the overall transport capacity of a mobile network. Further we derive a threshold value for the delivery error ratio, and conclude that no link state routing protocols can achieve a delivery error ratio smaller than this threshold.

\section{A. The Tradeoff between Control and Data Traffic}

Consider the design of link state routing protocols so as to ensure that the delivery ratio for data packets is no less than $(1-\eta)$. In other words, such protocols require each node to be able to compute a valid path for any possible destination with probability no less than $(1-\eta)$.

Let $\Psi_{\eta}$ denote the class of link state routing protocols capable of ensuring a delivery ratio of $(1-\eta)$. Then a lower 
bound on the routing overhead of any protocol $\psi \in \Psi_{\eta}$ is given by the following theorem.

Theorem 2: In order to ensure that the delivery ratio for data packets be no less than $(1-\eta)$, the lower bound on the minimum rate (in bits/sec) at which a node must receive control packets, denoted by $\tilde{\Phi}(\eta)$, is given by

$$
\begin{aligned}
\tilde{\Phi}(\eta) \geq & \frac{n(n-1)}{2 u}(h(Y)+h(Z) \\
& \left.+\log _{2} \frac{\ln \left(n-\left(\pi r^{2}\right)^{-1}\right)-\ln g(n, r)-1}{e^{2} \pi u^{2} r^{2}\left(\ln \left(n-\left(\pi r^{2}\right)^{-1}\right)-1\right) \cdot \eta}\right)
\end{aligned}
$$

where $g(n, r)$ is given by

$$
g(n, r)=n \ln \frac{1}{1-\pi r^{2}}
$$

Proof: Consider any protocol $\psi \in \Psi_{\eta}$. For any given nodes $k$ and $l$, let $\mathcal{A}_{t}$ denote the event that $k$ is able to compute a valid path to $l$ at time $t$, and let $\sigma(t)$ denote the probability that $\mathcal{A}_{t}$ happens, i.e.,

$$
\operatorname{Pr}\left[\mathcal{A}_{t}\right]=\sigma(t)
$$

Since protocol $\psi$ is able to route data packets with guaranteed delivery ratio of $(1-\eta)$, then we have

$$
\lim _{w \rightarrow \infty} \frac{1}{w} \int_{0}^{w} \sigma(t) d t \geq 1-\eta
$$

Let $\alpha_{1}(t)$ and $\alpha_{2}(t)$ denote the probabilities that type- 1 and type-2 errors in $(i, j)$ 's link state information occur at node $k$ at time $t$, respectively, where node $k$ and node pair $(i, j)$ are arbitrarily chosen. That is,

$$
\left\{\begin{array}{l}
\alpha_{1}(t)=\operatorname{Pr}[L(t)=0, \hat{L}(t)=1] \\
\alpha_{2}(t)=\operatorname{Pr}[L(t)=1, \hat{L}(t)=0]
\end{array}\right.
$$

Then the posteriori probability that link $(i, j)$ is down given that the perceived link status at node $k$ is up, denoted by $\zeta(t)$, is given by

$$
\begin{aligned}
\zeta(t) & =\operatorname{Pr}[L(t)=0 \mid \hat{L}(t)=1] \\
& =\frac{\operatorname{Pr}[L(t)=0, \hat{L}(t)=1]}{\operatorname{Pr}[L(t)=0, \hat{L}(t)=1]+\operatorname{Pr}[L(t)=1, \hat{L}(t)=1]} \\
& =\frac{\alpha_{1}(t)}{\alpha_{1}(t)+\pi r^{2}-\alpha_{2}(t)}
\end{aligned}
$$

Let $\bar{\alpha}_{1}, \bar{\alpha}_{2}$ and $\bar{\zeta}$ denote the time averages of $\alpha_{1}(t), \alpha_{2}(t)$ and $\zeta(t)$ respectively, i.e.,

$$
\left\{\begin{array}{l}
\bar{\alpha}_{1}=\lim _{w \rightarrow \infty} \frac{1}{w} \int_{0}^{w} \alpha_{1}(t) d t \\
\bar{\alpha}_{2}=\lim _{w \rightarrow \infty} \frac{1}{w} \int_{0}^{w} \alpha_{2}(t) d t \\
\bar{\zeta}=\lim _{w \rightarrow \infty} \frac{1}{w} \int_{0}^{w} \zeta(t) d t
\end{array}\right.
$$

Note that $\zeta(t) \geq \operatorname{Pr}[L(t)=0, \hat{L}(t)=1]=\alpha_{1}(t)$ and $\alpha_{2}(t) \leq \operatorname{Pr}[L(t)=1]=\pi r^{2}$, then it follows from (62) that

$$
\begin{aligned}
\bar{\zeta} & \geq \bar{\alpha}_{1} \\
\bar{\alpha}_{2} & \leq \pi r^{2}
\end{aligned}
$$

Let $S(t)$ denote the hop distance of the actual shortest path from $k$ to $l$ given the precise link state information of the network. Similarly let $\hat{S}(t)$ denote the hop distance of the computed path from $k$ to $l(\hat{S}(t):=\infty$ if $k$ is unable to compute a path to $l)$. Let $F_{S}(s):=\operatorname{Pr}[S>s]$ denote the complementary cumulative distribution function (ccdf) of $S(t)$, and let $F_{\hat{S}(t)}(s)$ denote the ccdf of $\hat{S}(t)^{1}$. Then the probability mass function (pmf) for $\hat{S}(t)$, denoted by $\left\{f_{\hat{S}(t)}(s)\right\}$, is given by

$$
f_{\hat{S}(t)}(s)=F_{\hat{S}(t)}(s-1)-F_{\hat{S}(t)}(s), \quad \forall S \in \mathbb{N} .
$$

According to Assumption 1, the probability that the computed path from $k$ to $l$ is a valid path given its hop distance $\hat{S}(t)=s$, denoted by $p_{s}(t)$, is given by

$$
p_{s}(t)=(\operatorname{Pr}[L(t)=1 \mid \hat{L}(t)=1])^{s}=(1-\zeta(t))^{s}
$$

Then the probability $\sigma(t)$ that $k$ is able to compute a valid path to $l$ at time $t$ is given by

$$
\begin{aligned}
\sigma(t) & =\sum_{s=1}^{n-1} f_{\hat{S}(t)}(s) p_{s}(t) \\
& =\sum_{s=1}^{\infty}(1-\zeta(t))^{s}\left(F_{\hat{S}(t)}(s-1)-F_{\hat{S}(t)}(s)\right) \\
& =1-\zeta(t) \sum_{s=0}^{\infty}(1-\zeta(t))^{s} F_{\hat{S}(t)}(s)
\end{aligned}
$$

Note that if node $k$ is able to compute a valid path from to $l$ at time $t$ (i.e. the event $\mathcal{A}_{t}$ happens), then obviously the hop distance of the computed path must be no less than the hop distance of the actual shortest path, i.e. $\hat{S}(t) \geq S(t)$. This fact implies that

$$
\operatorname{Pr}\left[\hat{S}(t)>s \mid \mathcal{A}_{t}\right] \geq \operatorname{Pr}\left[S(t)>s \mid \mathcal{A}_{t}\right]
$$

According to equation (58) and (68), we have

$$
\begin{aligned}
F_{\hat{S}(t)}(s) & \geq \operatorname{Pr}\left[\mathcal{A}_{t}\right] \operatorname{Pr}\left[\hat{S}(t)>s \mid \mathcal{A}_{t}\right] \\
& \geq \sigma(t) \operatorname{Pr}\left[S(t)>s \mid \mathcal{A}_{t}\right] \\
& \geq \sigma(t)\left(F_{S}(s)-\operatorname{Pr}\left[\overline{\mathcal{A}}_{t}\right] \operatorname{Pr}\left[S(t)>s \mid \overline{\mathcal{A}}_{t}\right]\right) \\
& =\sigma(t)\left(F_{S}(s)-(1-\sigma(t)) F_{S(t) \mid \overline{\mathcal{A}}_{t}}(s)\right)
\end{aligned}
$$

where $F_{S(t) \mid \overline{\mathcal{A}}_{t}}(s)=\operatorname{Pr}\left[S(t)>s \mid \overline{\mathcal{A}}_{t}\right]$ is the ccdf of $S(t)$ conditioned on the event $\overline{\mathcal{A}}_{t}$.

Note that $S$ is the hop distance of the shortest path between two vertices in a random graph in which there are $n$ vertices and each of the $\frac{n(n-1)}{2}$ possible edges occurs independently with probability $q:=\operatorname{Pr}[L(t)=1]=\pi r^{2}>\frac{1}{n}$. Then according to Lemma 3 in [21, Appendix], the ccdf of $S$ is lower bounded by

$$
F_{S}(s) \geq e^{-\frac{(g(n, r))^{s}}{n-\left(\pi r^{2}\right)^{-1}}}
$$

${ }^{1}$ Notice that the ccdf of $S(t)$ (i.e. $F_{S}(s)$ ) is time-invariant. This is implied by the fact that $S(t)$ is a stationary process. The stationarity of $S(t)$ can be deduced as follows. $S(t)$ is the hop distance of the shortest path from $k$ to $l$ at time $t$, which is determined by the node positions $\left\{X_{i}(t)\right\}_{i=1}^{n}$. Since $\left\{X_{i}(t)\right\}$ are assumed to be stationary and i.i.d., then $S(t)$ must also be a stationary process. 
It can be deduced from equation (67) and (69) that

$$
\begin{aligned}
\sigma(t) & \leq 1-\zeta(t) \sigma(t) \sum_{s=0}^{\infty}(1-\zeta(t))^{s} F_{S}(s) \\
& +\sigma(t)(1-\sigma(t)) \zeta(t) \sum_{s=0}^{\infty}(1-\zeta(t))^{s} F_{S(t) \mid \overline{\mathcal{A}}_{t}}(s)
\end{aligned}
$$

in which

$$
\begin{array}{r}
\sum_{s=0}^{\infty}(1-\zeta(t))^{s} F_{S(t) \mid \overline{\mathcal{A}}_{t}}(s) \leq \sum_{s=0}^{\infty}(1-\zeta(t))^{s}=\frac{1}{\zeta(t)} \\
\sum_{s=0}^{\infty}(1-\zeta(t))^{s} F_{H}(s) \geq \int_{0}^{\infty}(1-\zeta(t))^{s} e^{-\frac{(g(n, r))^{s}}{n-\left(\pi r^{2}\right)^{-1}}} d s \\
\geq \frac{n^{\frac{-\ln (1-\zeta(t))}{\ln g(n, r)}}}{\ln g(n, r)} \cdot \Gamma\left(\frac{\ln (1-\zeta(t))}{\ln g(n, r)}, \frac{1}{n-\left(\pi r^{2}\right)^{-1}}\right) \\
\geq \frac{1}{\ln g(n, r)} \cdot \Gamma\left(\frac{\ln (1-\zeta(t))}{\ln g(n, r)}, \frac{1}{n-\left(\pi r^{2}\right)^{-1}}\right)
\end{array}
$$

where $\Gamma(a, b)=\int_{b}^{\infty} x^{a-1} e^{-x} d x$ is the lower incomplete Gamma function. It is easy to show after some algebraic manipulations that

$$
\Gamma\left(\frac{\ln (1-\zeta(t))}{\ln g(n, r)}, \frac{1}{n}\right) \geq \ln \left(n-\left(\pi r^{2}\right)^{-1}\right)-1
$$

Combining (71), (72), (73) and (74) we get

$$
\sigma(t) \leq 1-\frac{\ln \left(n-\left(\pi r^{2}\right)^{-1}\right)-1}{\ln g(n, r)} \zeta(t) \sigma(t)+\sigma(t)-\sigma^{2}(t)
$$

Rearranging (75):

$$
\zeta(t) \leq \frac{\ln g(n, r)}{\ln \left(n-\left(\pi r^{2}\right)^{-1}\right)-1}\left(\frac{1}{\sigma(t)}-\sigma(t)\right)
$$

Since $0 \leq \zeta(t), \sigma(t) \leq 1$, then

$$
\begin{aligned}
\zeta(t) & \leq \min \left\{1, \frac{\ln g(n, r)}{\ln \left(n-\left(\pi r^{2}\right)^{-1}\right)-1}\left(\frac{1}{\sigma(t)}-\sigma(t)\right)\right\} \\
& \leq \frac{\ln \left(n-\left(\pi r^{2}\right)^{-1}\right)-1}{\ln \left(n-\left(\pi r^{2}\right)^{-1}\right)-\ln g(n, r)-1}(1-\sigma(t))
\end{aligned}
$$

Taking the time average of $\zeta(t)$ and then combining with (59):

$$
\begin{aligned}
\bar{\zeta} & \leq \lim _{w \rightarrow \infty} \frac{1}{w} \int_{0}^{w} \frac{\left(\ln \left(n-\left(\pi r^{2}\right)^{-1}\right)-1\right)(1-\sigma(t))}{\ln \left(n-\left(\pi r^{2}\right)^{-1}\right)-\ln g(n, r)-1} d t \\
& \leq \frac{\ln \left(n-\left(\pi r^{2}\right)^{-1}\right)-1}{\ln \left(n-\left(\pi r^{2}\right)^{-1}\right)-\ln g(n, r)-1} \cdot \eta
\end{aligned}
$$

Substituting (63) and (64) in (78) we have

$$
\bar{\alpha}_{1} \bar{\alpha}_{2} \leq \frac{\ln \left(n-\left(\pi r^{2}\right)^{-1}\right)-1}{\ln \left(n-\left(\pi r^{2}\right)^{-1}\right)-\ln g(n, r)-1} \cdot \pi r^{2} \eta
$$

According to Theorem 1, the bit rate of the minimum routing overhead is no less than $\Phi\left(\bar{\alpha}_{1}, \bar{\alpha}_{2}\right)$, i.e.,

$$
\tilde{\Phi}(\eta) \geq \Phi\left(\bar{\alpha}_{1}, \bar{\alpha}_{2}\right)
$$

Then the result follows directly after substituting (79) and (80) in (51).

The next corollary follows directly from Theorem 2 when Markovian link status changes are assumed.

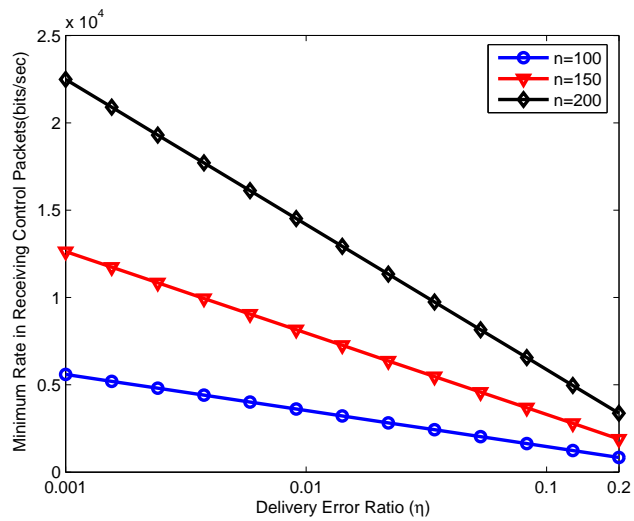

Fig. 4. The minimum rate of receiving control packets vs. delivery error ratio $\eta$ and the number of nodes in the network $n$.

Corollary 2: When the network is exhibiting Markovian link status changes, the lower bound on the minimum rate (in bits/sec) at which a node must receive control packets, while ensuring that the delivery ratio for data packets be no less than $(1-\eta)$, is given by

$$
\begin{aligned}
\tilde{\Phi}(\eta) & \geq \frac{n(n-1)}{2 u} . \\
& \log _{2} \frac{\left(1-\pi r^{2}\right)\left(\ln \left(n-\left(\pi r^{2}\right)^{-1}\right)-\ln g(n, r)-1\right)}{\left(\ln \left(n-\left(\pi r^{2}\right)^{-1}\right)-1\right) \cdot \eta}
\end{aligned}
$$

where $g(n, r)$ is given by equation (57)

Fig. 4 shows the plot of the lower bound on $\tilde{\Phi}$ against delivery error ratio $\eta$ and number of nodes $n$ according to (81). It is shown that for large $n$, the minimum rate at which a node must receive control packets becomes very high. Moreover, it is observed that there is a linear relationship between the lower bound on $\tilde{\Phi}$ and the logarithm of $\eta$, and $\tilde{\Phi}$ can be arbitrarily large with the decrease of $\eta$. This result characterizes the tradeoff between the routing overhead incurred by control traffic and the routing efficiency for data traffic.

\section{B. Capacity Deficit}

A wireless ad hoc network is said to transport one bit-meter when a bit is transmitted over a distance of one meter [4]. The transport capacity of a network (in bit-meters/sec) is defined as the supremum over the set of feasible rate vectors of the distance weighted sum of rates [20]. The transport capacity is expressed as $\lambda n \overline{\mathcal{L}}$, where $\lambda$ is the average arrival rate (in bits/sec) at the nodes, $n$ is the number of nodes and $\overline{\mathcal{L}}$ is the average distance travelled by the bits. It is shown in [4] that the transport capacity of an arbitrary wireless network is $\Theta(W \sqrt{n A})$, where $n, W$ and $A$ are the number of nodes deployed, the transmission rate of these nodes and the area over which the network is deployed respectively. It is shown in [4] that for a particular interference model known as the Protocol Model, the upper bound on the transport capacity of an arbitrary wireless network is given by

$$
\lambda n \overline{\mathcal{L}} \leq \frac{\sqrt{8}}{\pi} \frac{1}{\Delta} W \sqrt{n A}(\text { bit-meters/sec) }
$$


where $\Delta$ is some constant.

Theorem 2 shows that in order to ensure a delivery ratio as high as $(1-\eta)$, each node must receive control packets at the rate $\tilde{\Phi}(\eta)$ (in bits/sec). Since the a control packet received by a node travels at least one hop which covers a circular area of radius $r$, the total routing overhead measured in bit-meters/sec is at least $n r \tilde{\Phi}(\eta)$. This leads to the following theorem.

Theorem 3: For the Protocol Model, the upper bound on the residual transport capacity available to an arbitrary network for transmitting data $[\lambda n \overline{\mathcal{L}}]_{R}$ is given by

$$
[\lambda n \overline{\mathcal{L}}]_{R} \leq \frac{\sqrt{8}}{\pi} \frac{1}{\Delta} W \sqrt{n}-n r \tilde{\Phi}(\eta) \text { (bit-meters/sec) }
$$

Theorem 3 implies that it is impossible to make the delivery error ratio $\eta$ arbitrarily small, since the transport capacity may be overwhelmed by the overhead incurred by control packets. Combining Theorem 2 and 3 lead to the following corollary.

Corollary 3: For link state routing protocols, a delivery error ratio $\eta$ is not achievable if it is smaller than some threshold value $\eta^{\star}$. The lower bound on $\eta^{\star}$ is given by

$\eta^{\star} \geq\left(1-\frac{\ln g(n, r)}{\ln \left(n-\left(\pi r^{2}\right)^{-1}\right)-1}\right) \frac{2^{-\frac{4 \sqrt{2} W u}{\pi \Delta n^{3 / 2}(n-1) r}+h(Y)+h(Z)}}{e^{2} \pi u^{2} r^{2}}$

The next corollary follows directly when Markovian link status changes are assumed.

Corollary 4: When the network is exhibiting Markovian link status changes, for link state routing protocols, a delivery error ratio $\eta$ is not achievable if it is smaller than some threshold value $\eta^{\star}$. The lower bound on $\eta^{\star}$ is given by

$\eta^{\star} \geq\left(1-\frac{\ln g(n, r)}{\ln \left(n-\left(\pi r^{2}\right)^{-1}\right)-1}\right)\left(1-\pi r^{2}\right) \cdot 2^{\left(-\frac{4 \sqrt{2} W u}{\pi \Delta n^{3 / 2}(n-1) r}\right)}$

Fig. 5 shows the plot of the upper bound on $[\lambda n \overline{\mathcal{L}}]_{R}$ against delivery error ratio $\eta$ according to (83). It is worth noting that any $\left(\eta,[\lambda n \mathcal{\mathcal { L }}]_{R}\right)$ in the region above the curve cannot be achieved by any link state routing protocol.

\section{CONClusion And Future Work}

In this paper we presented an information-theoretic framework for analyzing the protocol overhead of link state routing in a mobile ad hoc network. We formulated the minimum overhead problem as a rate-distortion problem, and then derived lower bounds on the minimum bit-rate at which a node must receive link state information in order to route data packets with a guaranteed delivery ratio. This result is then connected with the results on the transport capacity of multi-hop wireless networks in [4] in order to characterize the effective capacity left for users. We derive a threshold value for the delivery error ratio, and conclude that no link state routing protocols can guarantee a delivery error ratio smaller than this threshold.

Directions of future research may include extending our proposed information-theoretic framework to a wider range of routing protocols. Developing practical routing schemes that approach to the bounds indicated by the theoretical results could be another option.

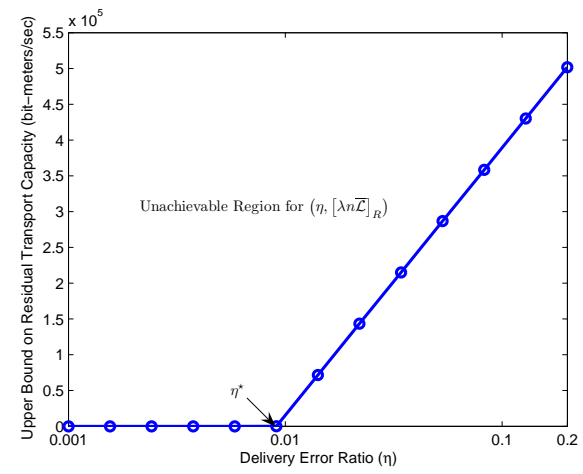

Fig. 5. The upper bound on the residual transport capacity available for transmitting data packets vs. delivery error ratio $\eta$.

\section{ACKNOWLEDGMENT}

This work was funded in part by the National Science Foundation under grants CNS-322956 and CNS-546402.

\section{REFERENCES}

[1] N. Zhou and A. Abouzeid, Routing in ad hoc networks: A theoretical framework with practical implications, Proc. INFOCOM'05, Mar. 2005.

[2] N. Bisnik and A. Abouzeid, Capacity deificit in mobile wirless ad hoc networks due to geographic routing overhead, Proc. INFOCOM'07, 2007.

[3] T. M. Cover and J. A. Thomas, Elements of Information Theory, $2 \mathrm{rd} \mathrm{ed}$. John Wiley \& Sons, 2005.

[4] P. Gupta and P. R. Kumar, The capacity of wireless networks, IEEE Trans. on Information Theory, pages 388 - 404, Mar. 2000.

[5] M. Grossglauser and D. N. C. Tse, Mobility increases the capacity of ad hoc wireless networks, IEEE/ACM Trans. on Networking, 10(4), 2002.

[6] G. Sharma, R. Mazumdar, and N. Shroff, Delay and capacity tradeoffs in mobile ad hoc networks: A global perspective, Proc. INFOCOM'06, 2006.

[7] J. Moy, OSPF version 2, IETF, RFC 1583, Mar., 1994.

[8] X. Hong, K. Xu and M. Gerla, Scalable routing protocols for mobile ad hoc networks, IEEE Network, 2002.

[9] A. Iwata et al., Scalable routing strategies for ad-hoc wireless networks, IEEE JSAC, Aug. 1999, pp. 1369C79.

[10] G. Pei, M. Gerla, and T. W. Chen, Fisheye state routing: A routing scheme for ad hoc wireless networks, Proc. ICC '00, Jun. 2000.

[11] C. Santivanez, R. Ramanathan, and I. Stavrakakis, Making link-state routing scale for ad hoc networks, Proc. ACM Intl. Symp. Mobile Ad Hoc Net. Comp., Oct. 2001.

[12] P. Jacquet et al., Optimized link state routing protocol, draft-ietfmanetolsr-05.txt, Internet Draft, IETF MANET Working Group, 2000.

[13] A. Qayyum, L. Viennot, and A. Laouiti, Multipoint relaying: An efficient technique for flooding in mobile wireless networks, PINRIA res. rep., RR3898, 2000.

[14] R. G. Ogier et al., Topology broadcast based on reverse-path forwarding (TBRPF), draft-ietf-manet-tbrpf-05.txt, Internet Draft, IETF MANET Working Group, Mar. 2002.

[15] R. G. Gallager, Basic limits on protocol information in data communication networks, IEEE Trans. on Inf. Theory, 22(4):385-398, 1976.

[16] Y. Han, R. J. La, A. M. Makowski and S. Lee, Distribution of path durations in mobile ad-hoc networks-Palm's Theorem to the rescue, Computer Networks, Volume 50, Issue 12, Network Modelling and Simulation, 24 August 2006, Pages 1887-1900.

[17] Q. M. Tran, A. Dadej, and S. Parreau, Characterizing mobility in ad hoc networks: A generalized approach, in Proc. of QWSN'05, 2005.

[18] G. Cheng and N. Ansari, Rate-distortion based link state update, Computer Networks, Volume 50, Issue 17, Dec. 2006.

[19] S. M. Ross, Stochastic Processes, 2rd ed. John Wiley \& Sons, 1996.

[20] F. Xue, L. L. Xie, and P. R. Kumar, The transport capacity of wireless networks over fading channels, IEEE Trans. on Inf. Theory, 51(3), 2005.

[21] D. Wang and A. Abouzeid, Link state routing overhead in mobile ad hoc networks: a rate distortion formulation, Technical Report, online: http://www.ecse.rpi.edu/homepages/abouzeid/link-state-overhead.pdf. 